\title{
GROWTH RATES AND CRITICAL EXPONENTS OF CLASSES OF BINARY COMBINATORIAL GEOMETRIES
}

\author{
BY
}

\author{
JOSEPH P. S. KUNG
}

\begin{abstract}
We prove that a binary geometry of rank $n(n \geqslant 2)$ not containing $M\left(K_{5}\right)$ and $F_{7}$ (respectively, $M\left(K_{5}\right)$ and $C_{10}$ ) as a minor has at most $3 n-3$ (respectively, $4 n-5$ ) points. Here, $M\left(K_{5}\right)$ is the cycle geometry of the complete graph on five vertices, $F_{7}$ the Fano plane, and $C_{10}$ a certain rank 4 ten-point geometry containing the dual Fano plane $F_{7}^{*}$ as a minor. Our technique is elementary and uses the notion of a bond graph. From these results, we deduce upper bounds on the critical exponents of these geometries.
\end{abstract}

1. Introduction. If a binary combinatorial geometry (or simple matroid) of rank $n$ does not contain certain geometries as minors, how many points (as a function of $n$ ) can it have? In this paper, we answer this question for binary geometries not containing (a) $M\left(K_{5}\right)$ and $F_{7}$, and (b) $M\left(K_{5}\right)$ and $C_{10}$, where $C_{10}$ is a binary geometry with 10 points containing $F_{7}^{*}$ as a subgeometry. Our approach is elementary. It uses the notion of a bond graph which gives a way of visualising the incidence relations of lines in a binary geometry relative to a copoint. From our main results, we deduce upper bounds on the critical exponents of these geometries.

We shall assume that the reader is familiar with the theory of combinatorial geometries or matroids as set forth in $[4,17$, or 18]. To clarify our terminology, let $G$ be a finite geometric lattice. If $S$ is the set of points (or rank one flats) in $G$, the lattice structure of $G$ induces the structure of a geometry, also denoted by $G$, on $S$. The size $|G|$ of the geometry $G$ is the number of points in $G$. Let $T$ be a subset of $S$. The deletion of $T$ from $G$ is the geometry on the point set $S \backslash T$ obtained by restricting $G$ to the subset $S \backslash T$. The contraction $G / T$ of $G$ by $T$ is the geometry induced by the geometric lattice $[\bar{T}, \hat{1}]$ on the set $S^{\prime}$ of all flats in $G$ covering $\bar{T}$. (Here, $\bar{T}$ is the closure of $T$, and $\hat{1}$ is the maximum of the lattice $G$.) Thus, by definition, the contraction of a geometry is always a geometry-indeed, it is isomorphic to the simplification of the matroid $G / T$ as defined, say, in [17, p. 62]. A geometry which can be obtained from $G$ by deletions and contractions is called a minor of $G$.

Received by the editors April 8, 1985. Presented at the Special Session on the Structure of Graphs and Matroids at the American Mathematical Society Summer Meeting in Eugene, Oregon, August, 1984.

1980 Mathematics Subject Classification. Primary 05B35; Secondary 05C35.

Kev words and phrases. Matroid theory, minor-closed classes, growth rates, critical exponents, bond graphs. 
For ready reference, we note here several elementary facts about binary geometries. We shall often regard a binary geometry as a set of nonzero vectors over $\mathrm{GF}(2)$.

(1.1) A three-point line in a binary geometry is modular. In particular, it is either contained in a copoint or intersects it at exactly one point.

(1.2) A binary geometry is affine (i.e. contained in the complement of a hyperplane) if and only if all its circuits have even cardinality at least 4 . An affine binary geometry of rank $n$ has at most $2^{n-1}$ points.

(1.3) Let $\{a, b, c, d\}$ and $\{a, b, c, e\}$ be subsets of points in a binary affine geometry. Then at least one of these subsets is independent.

The proofs of (1.1) and (1.2) are standard. To prove (1.3), suppose that both $\{a, b, c, d\}$ and $\{a, b, c, e\}$ are dependent. By (1.2), they are both circuits. As symmetric differences of circuits are dependent in a binary geometry, $\{d, e\}$ is dependent, contradicting our assumption that $G$ is a geometry.

We turn now to graphs. A graph $\Delta$ is said to be a subgraph of the graph $\Gamma$ if the vertex and edge set of $\Delta$ are subsets of the vertex and edge set of $\Gamma$. If $Q$ is a subset of vertices of $\Gamma$, the subgraph $\Gamma \mid Q$ induced by $Q$ is the graph on the vertex set $Q$ and edge set all the edges of $\Gamma$ with both endpoints in $Q$. A subgraph $\Delta$ is an edge subgraph of $\Gamma$ if the vertex set of $\Delta$ equals the vertex set of $\Gamma$. Let $\Gamma$ and $\Delta$ be graphs. Then $\Delta$ is said to be a topological $\Gamma$ if $\Delta$ can be obtained from $\Gamma$ by subdividing edges. The vertices in $\Delta$ which are also vertices in $\Gamma$ are called base vertices. If $\Gamma$ is a graph, we denote by $M(\Gamma)$ its cycle geometry.

Finally, we remark that we shall always use the word "connected" when applied to a graph to mean connected as a graph, the word "circuit" to mean a minimal dependent set in a geometry, and the word "cycle" to mean a simple closed path in a graph.

2. Minor-closed classes and their growth rates. A class of geometries is a collection of geometries closed under isomorphism. A class $\mathscr{C}$ is said to be minor-closed if $G \in \mathscr{C}$ and $H$ is a minor of $G \Rightarrow H \in \mathscr{C}$. Examples of minor-closed classes are readily obtained by excluding certain geometries as minors. More precisely, let $\left\{M_{\alpha}\right\}$ be a (possibly infinite) set of geometries. The class

$\mathscr{E} x\left(M_{\alpha}\right)=\left\{G: G\right.$ does not contain any of the geometries $M_{\alpha}$ as minors $\}$

is obviously a minor-closed class. Conversely, any minor-closed class is of this form - simply exclude all the geometries not in it!

Let $\mathscr{C}$ be a class of geometries. The size function $h(\mathscr{C}, n)$, or briefly, $h(n)$, of $\mathscr{C}$ is the partial function defined on the nonnegative integers by

$$
h(\mathscr{C}, n)=\max \{|G|: G \in \mathscr{C} \text { and } \operatorname{rank}(G)=n\}
$$

if $\mathscr{C}$ contains a geometry of rank $n$ and the maximum exists; it is undefined otherwise.

Often, it is more convenient to work with the growth rate $g(\mathscr{C}, n)$, or briefly, $g(n)$, of $\mathscr{C}$ defined by

$$
g(\mathscr{C}, n)=h(\mathscr{C}, n)-h(\mathscr{C}, n-1)
$$


The maximum growth rate $g(\mathscr{C})$ is the integer $\max \{g(n, \mathscr{C}): 1 \leqslant n<\infty\}$, if it exists, and is said to be infinite otherwise.

As we shall be concerned exclusively with binary geometries in this paper, we shall adopt the convention that $\mathscr{E} x\left(M_{\alpha}\right)$ is the class of binary geometries not containing $M_{\alpha}$ as minors; that is, by Tutte's characterization of binary geometries, we implicitly add $U_{4}^{2}$, the four-point line, to our list of excluded minors.

In order to state our main results, we need to describe four binary geometries. The first three are quite familiar, but the fourth makes its debut here.

(2.1) $M\left(K_{5}\right)$. This is the cycle geometry of $K_{5}$, the complete graph on five vertices. We shall often identify this geometry with its natural representation as the 10-element set of all binary four-dimensional vectors with exactly one or exactly two nonzero coordinates.

(2.2) $F_{7}$. This is the Fano plane and can be represented by the seven-element set of all nonzero binary three-dimensional vectors.

(2.3) $F_{7}^{*}$. This is the orthogonal dual of $F_{7}$. It is of rank 4 and can be represented by any seven four-dimensional vectors in affine binary space of rank 4 (or dimension 3).

(2.4) $C_{10}$. This is most easily described as the binary geometry obtained from the natural representation of $M\left(K_{5}\right)$ by removing the vector $(0,1,1,0)$ and replacing it with $(1,1,1,0)$. It is, of course, a nongraphic geometry. $C_{10}$ contains $F_{7}^{*}$ as a subgeometry. To see this, observe that the three vectors $(0,1,0,0),(0,0,1,0)$, and $(1,0,0,1)$ are independent, and their linear span contains no other vector in $C_{10}$. Hence, they form a copoint of size 3. The complement of this copoint is an affine geometry of rank 4 containing seven points and is isomorphic to $F_{7}{ }^{*}$.

The main results can now be stated.

(2.5) THEOREM. Let $g(n)$ be the growth rate of $\mathscr{E} x\left(M\left(K_{5}\right), F_{7}\right)$. Then for $n \geqslant 3$, $g(n)=3$.

(2.6) THEOREM. Let $g(n)$ be the growth rate of $\mathscr{E} x\left(M\left(K_{5}\right), C_{10}\right)$. Then for $n \geqslant 3$, $g(n)=4$.

The proofs of these theorems use the notion of a bond graph, the subject of $\S 4$.

From (2.4), (2.6), and the fact that $\mathscr{E} x\left(M\left(K_{5}\right), F_{7}{ }^{*}\right)$ contains $F_{7}$, we obtain

(2.7) COROllaRY. The maximum growth rate of $\mathscr{E} x\left(M\left(K_{5}\right), F_{7}^{*}\right)$ is 4 .

This is not the most precise result possible. Using Seymour's decomposition theory, we shall obtain the precise growth rate of $\mathscr{E} x\left(M\left(K_{5}\right), F_{7}{ }^{*}\right)$ in $\S 9$.

3. Uniform critical exponents. In this section, we explore the connection between growth rates and the critical problem. Let $G$ be a geometry representable over the finite field $\operatorname{GF}(q)$ and choose a representation of $G$ as a set of vectors in a finite dimensional vector space $V$ over $\operatorname{GF}(q)$. The critical exponent $e(G)$ is the smallest positive integer for which there exist $e(G)$ hyperplanes $H_{1}, H_{2}, \ldots, H_{e(G)}$ of $V$ such that

$$
H_{1} \cap H_{2} \cap \cdots \cap H_{e(G)} \cap G=\varnothing .
$$


Equivalently, $e(G)$ is the smallest integer such that $G$ is the union of $e(G)$ affine subgeometries. The critical exponent does not depend on the choice of representation of $G$ as a set of vectors, only on $q$, the order of the field $\operatorname{GF}(q)$, and the structure of $G$ as a geometry. For further details, see [4, Chapter 16 or 17, p. 273].

If $\mathscr{C}$ is a class of geometries representable over $\operatorname{GF}(q)$, its uniform critical exponent $e(\mathscr{C})$ is defined by

$$
e(\mathscr{C})=\max \{e(G): G \in \mathscr{C}\}
$$

if the maximum exists (and is finite), and is infinite if the maximum does not exist. The uniform critical exponent and growth rate are related by the following result.

(3.1) Lemma. Let $\mathscr{C}$ be a class of geometries representable over $\mathrm{GF}(q)$ closed under subgeometries (only!). Suppose its size function $h(n)$ satisfies $h(n) \leqslant c n$ for some integer $c$. Then, $e(\mathscr{C}) \leqslant c$. In other words, the uniform critical exponent is at most the maximum growth rate.

Proof. The technique used is a direct generalization of Jaeger's proof of the eight-flow theorem [9, proof of Proposition 8, p. 211]. Let $G$ be a geometry in $\mathscr{C}$. Since $\mathscr{C}$ is closed under subgeometries, we have, for all subgeometries $H$ of $G$, $|H| \leqslant \operatorname{crank}(H)$. By the matroid partition theorem [7 or 17, p. 365], $G$ can be partitioned into $c$ independent sets. Since independent sets are affine, $G$ is the union of $c$ affine subgeometries. Hence, $e(G) \leqslant c$.

From this lemma and our earlier results on growth rates, we obtain

(3.2) THEOREM. (a) $e\left(\mathscr{E} x\left(M\left(K_{5}\right), F_{7}\right)\right)=3$.

(b) $e\left(\mathscr{E} x\left(M\left(K_{5}\right), C_{10}\right)\right)=3$ or 4 .

(c) $e\left(\mathscr{E} x\left(M\left(K_{5}\right), F_{7}^{*}\right)\right)=3$ or 4 .

Proof. The upper bounds follow from (3.1), (2.5), (2.6), and (2.7). Equality in (a) follows from the existence of a cographic geometry (e.g. the cocycle geometry of the Petersen graph) with critical exponent 3. Similarly, the lower bounds in (b) and (c) are attained by $F_{7}$.

In fact, as was shown by Walton and Welsh [16], $e\left(\mathscr{E} x\left(M\left(K_{5}\right), F_{7}^{*}\right)\right)=3$. In addition, they also proved that $e\left(\mathscr{E} x\left(M\left(K_{5}\right), F_{7}\right)\right)=3$. Their proof uses Seymour's decomposition theory (cf. $\S 9$ ) and does not shed any light on the exact value of $e\left(\mathscr{E} x\left(M\left(K_{5}\right), C_{10}\right)\right)$.

We end with a conjecture.

(3.3) CONJECTURE. Let $\mathscr{C}$ be a minor-closed class of geometries representable over $\operatorname{GF}(q)$. Then the uniform critical exponent is finite if and only if the maximum growth rate is finite.

4. Bond graphs. Let $G$ be a binary geometry, and let $x$ be a copoint of $G$. The bond graph $\Gamma(x, G)$ is the labelled graph defined as follows. The vertex set of $\Gamma(x, G)$ is the set of points in $G$ not contained in the copoint $x$. Two vertices $a$ and $b$ are joined by an edge $\{a, b\}$ if there exists a third point $\gamma$ on the line $a \vee b$ in th 
geometry $G$. By (1.1), the third point $\gamma$, if it is in $G$, is uniquely determined by $a$ and $b$ and is necessarily in the copoint $x$. We label the edge $\{a, b\}$ by $\gamma$ and use the notation $l(a, b)=\gamma$.

We first derive several simple consequences of the definition.

(4.1) Lemma. Let $\Gamma(x, G)$ be a bond graph.

(a) Let $C$ be a subset of vertices of $\Gamma(x, G)$ forming a circuit of $G$. Then $|C|$ is even and at least 4.

(b) Let $\{a, b\},\{b, c\}, \ldots,\{d, e\}$ be a simple path in $\Gamma(x, G)$, and let $L$ be the set of edge labels appearing an odd number of times in the list $l(a, b), l(b, c), \ldots, l(d, e)$. Then the set of points $L \cup\{a, e\}$ is a disjoint union of circuits in $G$. In particular, $e$ is in the closure of $L \cup\{a\}$.

(c) Let $\{a, b\},\{b, c\}, \ldots,\{d, a\}$ be a cycle in $\Gamma(x, G)$, and let $L$ be the set of edge labels appearing an odd number of times in the list $l(a, b), l(b, c), \ldots, l(d, a)$. Then $L$ is a disjoint union of circuits of $G$.

(d) Two incident edges cannot have the same label.

Proof. The vertex set of $\Gamma(x, G)$ is contained in the complement of the hyperplane spanned by the copoint $x$. Hence, it is an affine subgeometry of $G$. The first assertion now follows from (1.2).

The second assertion follows from taking the symmetric differences of the circuits $\{a, l(a, b), b\},\{b, l(b, c), c\}, \ldots,\{d, l(d, e), e\}$. The third assertion follows similarly.

To prove the fourth assertion, observe that if $\{a, b\}$ and $\{a, c\}$ are edges and $l(a, b)=l(a, c)$, then by $(\mathrm{b})\{b, c\}$ is a circuit, contradicting (a).

The next lemma is quite easy but turns out to be very useful.

(4.2) Lemma. If $a, b, c, d$ are coplanar and $\{a, b\}$ is an edge, then $\{c, d\}$ is an edge labelled by $l(a, b)$. In particular, if $\{a, b\},\{c, d\}$, and $\{a, c\}$ are edges and $l(a, b)=l(c, d)$, then $\{b, d\}$ is an edge labelled by $l(a, c)$.

We next study the behaviour of bond graphs under deletion and contraction.

(4.3) Lemma. Let $E$ be a subset of vertices of the bond graph $\Gamma(x, G)$. If the induced subgraph $\Gamma(x, G) \mid E$ is connected, then it is also a bond graph.

Proof. Let $u$ be the set of points in $x$ occurring as the label of an edge with both endpoints in $E$, and let $H$ be the subgeometry of $G$ consisting of the points in $E \cup u$. We claim that $\Gamma(x, G) \mid E$ equals the bond graph $\Gamma(u, H)$. As it is evident that $u$ is closed in $H$, it suffices to show that $r(u)=r(H)-1$. Let $a$ be any vertex in $E$. Since $\Gamma(x, G) \mid E$ is connected, there exists a simple path from $a$ to any other vertex in $E$. By (4.1)(b), the set $u \cup\{a\}$ spans $H$. From this we deduce that $r(u)=r(H)-1$.

A similar argument proves

(4.4) Lemma. Let $\alpha$ be an edge label occurring in $\Gamma(x, G)$. Suppose that the graph $\Gamma(x, G) \backslash \backslash \alpha$ from $\Gamma(x, G)$ by deleting all the edges labelled by $\alpha$ is connected. Then $\Gamma(x, G) \backslash \backslash \alpha$ is a bond graph. 
REMARK. The collection of bond graphs is not closed under deletions. This may be remedied by defining bond graphs using generalized copoints. Let $x$ be a flat of a binary geometry $G$ represented as a set of vectors in $[\mathrm{GF}(2)]^{n}$. Then $x$ is said to be a generalized copoint of $G$ if $x$ equals the intersection of $G$ with a hyperplane in $[\mathrm{GF}(2)]^{n}$. Since representations of a binary geometry are projectively equivalent $[3$, p. 93], this definition is independent of the representation chosen. The theory of bond graphs works in this more general setting. However, this extra generality is never needed in applications.

Now, let $\alpha$ be a point in the copoint $x$. The set

$$
x / \alpha=\{p \vee \alpha: p \text { is a point and } p \leqslant x\}
$$

of all lines in $G$ covering $\alpha$ and contained in $x$ is a copoint in the contraction $G / \alpha$. How is the bond graph $\Gamma(x / \alpha, G / \alpha)$ related to the bond graph $\Gamma(x, G)$ ?

To answer this question, let $\Gamma(x, G) / / \alpha$ be the graph obtained from $\Gamma(x, G)$ by graphically contracting all the edges labelled by $\alpha$ and replacing all multiple edges by single edges. Two vertices $b$ and $c$ are identified in $\Gamma(x, G) / / \alpha$ if they are joined by an edge labelled $\alpha$. Observe that

$$
\begin{aligned}
& \{b, c\} \text { is an edge labelled by } \alpha \\
& \quad \Leftrightarrow \alpha, b, c \text { is collinear in } G \\
& \quad \Leftrightarrow \text { the lines } b \vee \alpha \text { and } c \vee \alpha \text { are equal. }
\end{aligned}
$$

In addition, observe that since $b \nless x$, the line $b \vee \alpha$ is not contained in $x / \alpha$. From these observations, we conclude that the vertices in $\Gamma(x, G) / / \alpha$ can be naturally identified with the vertices of $\Gamma(x / \alpha, G / \alpha)$.

We next consider the edges of $\Gamma(x, G) / / \alpha$. Let $\{b \vee \alpha, c \vee \alpha\}$ be an edge in $\Gamma(x, G) / / \alpha$. Since $\Gamma(x, G)$ is obtained by contracting edges labelled by $\alpha$, there exist points $b^{\prime} \leqslant b \vee \alpha$ and $c^{\prime} \leqslant c \vee \alpha$ such that $\left\{b^{\prime}, c^{\prime}\right\}$ is an edge in $\Gamma(x, G)$. We label $\{b \vee \alpha, c \vee \alpha\}$ by the line $l\left(b^{\prime}, c^{\prime}\right) \vee \alpha$. We need to check that this labelling is consistent.

Suppose that $b^{\prime \prime} \leqslant b \vee \alpha$ and $c^{\prime \prime} \leqslant c \vee \alpha$ are two other points such that $\left\{b^{\prime \prime}, c^{\prime \prime}\right\}$ is an edge in $\Gamma(x, G)$. First consider the case when $b^{\prime} \neq b^{\prime \prime}$ and $c^{\prime} \neq c^{\prime \prime}$. As $b^{\prime}, b^{\prime \prime}$, $\alpha$ and $c^{\prime}, c^{\prime \prime}, \alpha$ are collinear, $\left\{b^{\prime}, b^{\prime \prime}\right\}$ and $\left\{c^{\prime}, c^{\prime \prime}\right\}$ are edges in $\Gamma(x, G)$ labelled by $\alpha$. The edges $\left\{b^{\prime}, c^{\prime}\right\},\left\{c^{\prime}, c^{\prime \prime}\right\},\left\{c^{\prime \prime}, b^{\prime \prime}\right\},\left\{b^{\prime \prime}, b^{\prime}\right\}$ form a cycle in $\Gamma(x, G)$. The list of edge labels in this cycle is $l\left(b^{\prime}, c^{\prime}\right), \alpha, \alpha, l\left(b^{\prime \prime}, c^{\prime \prime}\right)$. Hence, by $(4.1)(c), l\left(b^{\prime}, c^{\prime}\right)=$ $l\left(b^{\prime \prime}, c^{\prime \prime}\right)$. It remains to consider the case when, say, $c^{\prime}=c^{\prime \prime}$ but $b^{\prime} \neq b^{\prime \prime}$. The edges $\left\{b^{\prime}, c^{\prime}\right\},\left\{c^{\prime}, b^{\prime \prime}\right\},\left\{b^{\prime \prime}, b^{\prime}\right\}$ form a graphical triangle in $\Gamma(x, G)$. By $(4.1)(\mathrm{c})$ and $(4.1)(\mathrm{d})$, the edge labels $l\left(b^{\prime}, c^{\prime}\right), l\left(c^{\prime}, b^{\prime \prime}\right), \alpha$ form a circuit in $G$ and are collinear. Thus, $l\left(b^{\prime}, c^{\prime}\right) \vee \alpha=l\left(b^{\prime \prime}, c^{\prime}\right) \vee \alpha$. We conclude that our labelling is independent of the choice of the points $b^{\prime}$ and $c^{\prime}$. To summarize, we have proved the following lemma.

(4.5) LeMMA. $\Gamma(x, G) / / \alpha$ is an edge subgraph of $\Gamma(x / \alpha, G / \alpha)$.

This result is the best possible. One can easily construct examples where $\Gamma(x / \alpha, G / \alpha)$ has more edges than $\Gamma(x, G) / / \alpha$. 
Our final result relates the minimum degree in a bond graph of a geometry of maximum size in a minor-closed class to the growth rate.

(4.6) LeMma. Let $\mathscr{C}$ be a minor-closed class of binary geometries with size function $h(n)$ and growth rate $g(n)$. Let $G$ be a geometry in $\mathscr{C}$ of rank $n$ and maximum size $h(n)$, and $x$ a copoint in $G$. Then every vertex of the bond graph $\Gamma(x, G)$ has degree at least $g(n)-1$.

Proof. Let $a$ be any point in the complement of $x$. The contraction $G / a$ is a geometry of rank $n-1$ in $\mathscr{C}$. Hence, $G / a$ has at most $h(n-1)$ points; that is, there are at most $h(n-1)$ lines in $G$ covering the point $a$. Thus, there must be at least

$$
[h(n)-1]-h(n-1)=g(n)-1
$$

three-point lines covering $a$. In each of these three-point lines, $a$ and another point are not in $x$, and the third point is in $x$ (see (1.1)). We conclude that the vertex $a$ is joined to at least $g(n)-1$ other vertices in $\Gamma(x, G)$.

5. Series-parallel networks. The proofs of the main theorems, although simple in structure, require the consideration of many cases. In order to clarify the basic ideas, it may be worthwhile to consider as a test case the simpler (but still nontrivial) problem of determining the maximum growth rate of $\mathscr{E} x\left(M\left(K_{4}\right)\right)$. Naturally, this section may be skipped by the impatient reader.

(5.1) THEOREM. The growth rate $g(n)$ of $\mathscr{E} x\left(M\left(K_{4}\right)\right)$ equals 2 for $n \geqslant 2$.

PROOF USING THE BOND GRAPH. We first show that the maximum growth rate of $\mathscr{E} x\left(M\left(K_{4}\right)\right)$ is at most 2 .

(5.2) Proposition. Let $\mathscr{C}$ be a minor-closed class of binary geometries with maximum growth rate at least 3 . Then $\mathscr{C}$ contains $M\left(K_{4}\right)$.

The proof of (5.2) consists of three main steps. The first step is to observe that, by (4.6), there exists a bond graph $\Gamma(x, G)$ with $G$ in $\mathscr{C}$ such that every vertex in $\Gamma(x, G)$ has degree at least 2 .

In the second step, we recall an (almost obvious) fact from extremal graph theory.

(5.2.1) Let $\Gamma$ be a graph in which every vertex has degree at least 2 . Then $\Gamma$ contains a cycle.

From this, we conclude that there exists a bond graph $\Gamma(x, G)$ with $G$ in $\mathscr{C}$ containing a cycle.

In the third step, we consider, among all such bond graphs containing cycles, bond graphs $\Gamma(x, G)$ which are minimal in the sense that $\Gamma(x, G)$ contains the smallest possible number of vertices and edges.

(5.2.2) A minimal bond graph $\Gamma(x, G)$ is either

(a) a cycle with three vertices, or

(b) a cycle with four vertices $a, b, c, d$, and edges $\{a, b\},\{b, c\},\{c, d\},\{d, a\}$, such that $l(a, b)=l(c, d)$ and $l(b, c)=l(d, a)$. 
Proof. By minimality, $\Gamma(x, G)$ is a cycle; otherwise, by (4.3) or (4.4), vertices or edges can be deleted. Let $\Gamma(x, G)$ be the cycle on the vertices $a_{0}, a_{1}, \ldots, a_{n-1}$ with edges $\left\{a_{i}, a_{i+1}\right\}$, the subscripts being taken modulo $n$. Suppose that $n \geqslant 5$. Then, as (i) $l\left(a_{i}, a_{i+1}\right) \neq l\left(a_{i+1}, a_{i+2}\right)$ (by (4.1)(d)) and (ii) $l\left(a_{i}, a_{i+1}\right) \neq l\left(a_{i+2}, a_{i+3}\right)$ (otherwise, by (4.2), $\left\{a_{i}, a_{i+3}\right\}$ is an edge, contradicting $\left.n \geqslant 5\right)$, there are at least three distinct edge labels in $\Gamma(x, G)$. Thus, if $\alpha$ is an edge label, the graph $\Gamma(x, G) / / \alpha$ is still a cycle. Hence, by $(4.5), \Gamma(x / \alpha, G / \alpha)$ is a bond graph containing a cycle with fewer vertices than $\Gamma(x, G)$, contradicting minimality.

We conclude that $n=3$ or 4 . If $n=3$, then (a) holds. If $n=4$ but the edges are labelled by distinct labels, then one of the edge labels can be contracted to yield a cycle with three vertices, contradicting minimality. Hence, two edges, which by (4.2) are not incident, have the same label. The other two edges must also have the same label by (4.2). Thus, (b) holds when $n=4$.

To finish the proof, we shall show that if $\Gamma(x, G)$ is minimal, then $G$ is isomorphic to $M\left(K_{4}\right)$. If (a) holds, then $\Gamma(x, G)$ is a cycle, with vertices $a, b, c$ and edges $\{a, b\},\{b, c\}\{c, a\}$. By $(4.1)(\mathrm{a}),\{a, b, c\}$ is independent. Setting $a=(1,0,0)$, $b=(0,1,0)$, and $c=(0,0,1)$, we see that $a, b, c$, together with the edge labels, form a $M\left(K_{4}\right)$. If (b) holds, then $a, b, c, d$ are coplanar by (4.2). Setting $a=(1,0,0)$, $l(a, b)=(0,1,0)$, and $l(a, d)=(0,0,1)$, we see that the six points occurring as vertex or edge labels in $\Gamma(x, G)$ form a $M\left(K_{4}\right)$.

To finish the proof of (5.1), observe that the cycle geometries of series-parallel networks do not contain $M\left(K_{4}\right)$ as a minor and attain the maximum growth rate of 2.

Alternative Proof. (5.1) can also be proved by using known but nonelementary results.

First observe that $M\left(K_{4}\right)$ is contained as a minor in $F_{7}, F_{7}^{*}, M\left(K_{5}\right)^{*}$, and $M\left(K_{3,3}\right)^{*}$. Thus, by Tutte's characterization of graphic geometries [15 or 17, p. 176], every geometry in $\mathscr{E} x\left(M\left(K_{4}\right)\right)$ is graphic. (5.1) now follows from Dirac's theorem [5, p. 68 or 1, p. 379] stating that a graph on $n$ vertices not containing $K_{4}$ as a subcontraction has at most $2 n-3$ edges. Alternatively, we can use the related theorem of Duffin [6], stating that a graph not containing $M\left(K_{4}\right)$ as a subcontraction is a series-parallel network, and verify that a series-parallel network with no multiple edges on $n$ vertices can have at most $2 n-3$ edges.

6. Two results from extremal graph theory. In $\S 8$, we shall need two results from extremal graph theory. These results guarantee the existence of certain topological subgraphs in a graph provided that the minimum degree is sufficiently high. The first result is a classic theorem of Dirac [5, p. 67].

(6.1) TheOREM. Let $\Gamma$ be a graph with minimum degree at least 3. Then $\Gamma$ contains a topological $K_{4}$ as a subgraph.

The second result (which we shall not state in its full generality) is due to Pelikán [11]. (See also [13 or 1, p. 381].) 
(6.2) THEOREM. Let $\Gamma$ be a graph with minimum degree at least 4. Then $\Gamma$ contains a topological $K_{5}^{-}$as a subgraph. Here, $K_{5}^{-}$is the graph obtained from $K_{5}$, the complete graph on 5 vertices, by removing an edge.

7. Some small binary geometries. The following lemma about binary geometries of rank 4 will prove to be very useful in the next section.

(7.1) Lemma. Let $\Gamma(x, G)$ be a bond graph such that

(a) $\Gamma(x, G)$ contains a topological $K_{4}$ whose base vertices are independent, and

(b) $G$ is of rank 4.

Then $G$ contains $M\left(K_{5}\right)$ or $C_{10}$ as a subgeometry.

One way to prove this is to enumerate all the possibilities, of which there are only a finite number. A more satisfying way proceeds via the next three lemmas.

(7.2) Lemma. Under the hypotheses of (7.1), G contains at least 10 points.

ProOF. Let $a$ and $b$ be two base vertices of the topological $K_{4}$ contained in $\Gamma(x, G)$. Consider the path between $a$ and $b$ in the topological $K_{4}$. If this path is not the edge $\{a, b\}$, then each vertex inside the path contributes one point to $G$. If $\{a, b\}$ is an edge, then its label is distinct from the label of any edge $\{u, v\}$, where both $u$ and $v$ are base vertices. (This follows from (4.1)(d) if $\{a, b\}$ and $\{u, v\}$ are incident. If not, then $a, b, u, v$ are the four base vertices and are independent; hence, the edge labels are distinct by (4.2).) Thus, each of the six paths between two base vertices in the topological $K_{4}$ contributes at least one distinct point to $G$. These points, together with the four base vertices, give a total of at least 10 points.

A geometry $G$ is said to split if there exist two proper flats $X$ and $Y$ such that (as set of points) $G=X \cup Y$.

(7.3) LeMma. Under the hypotheses of (7.1), G does not split.

Proof. Suppose that $G=X \cup Y$, where $X$ and $Y$ are flats of rank at most 3.

(7.3.1) Let $u$ and $v$ be vertices such that $u \in X, v \in Y$, but $u, v \notin X \cap Y$. Then $\{u, v\}$ is not an edge.

Proof. As $G=X \cup Y$, the edge label $l(u, v)$ is in $X$ or $Y$. By symmetry, we may as well assume that it is in $X$. But, as $u, l(u, v), v$ are collinear, $v$ is also in $X$, contradicting the last hypothesis.

(7.3.2) There are at least three vertices in $X \cap Y$.

Proof. As the base vertices of the topological $K_{4}$ are independent and form a subset of rank 4 , neither $X$ nor $Y$ contains all the base vertices. Thus, as is easy to check, there are at least three vertex disjoint paths $P_{1}, P_{2}$, and $P_{3}$ starting at a base vertex in $X$ and ending in a base vertex in $Y$. Thinking of the path $P_{i}$ as a sequence of vertices, let $u_{i}$ be the first vertex in the path to be in $Y$. We claim that $u_{i}$ is also in $X$. To see this, observe first that if $u_{i}$ is in fact the first vertex in $P_{i}$, then our claim holds trivially. Now suppose that $u_{i}$ is not in $X$. Let $v_{i}$ be the vertex in $P_{i}$ occurring immediately before $u_{i}$ in $P_{i}$. By our choice of $u_{i}, v_{i}$ is in $X$ but not in $Y$, while $u_{i}$ is in $Y$ but not in $X$. However, $\left\{v_{i}, u_{i}\right\}$ is an edge, contradicting (7.3.1). This proves the claim. The three vertices $u_{1}, u_{2}$, and $u_{3}$ are in $X \cap Y$. 
Since neither $X$ nor $Y$ contain all the vertices but their union does, we can find a vertex $s$ in $X$ but not in $Y$ and a vertex $t$ in $Y$ but not in $X$. Now, $X$ and $Y$ are of rank at most 3 , and so $\left\{u_{1}, u_{2}, u_{3}, s\right\}$ and $\left\{u_{1}, u_{2}, u_{3}, t\right\}$ are both dependent. But this is impossible by (1.3). Hence, $G$ does not split.

The proof of (7.1) is completed by checking the following lemma.

(7.4) Lemma. A binary geometry of rank 4 which has at least 10 points and does not split contains $M\left(K_{5}\right)$ or $C_{10}$ as a subgeometry.

Proof. A binary geometry $G$ of rank 4 with at least 10 points can be obtained by removing a set $G^{c}$ of at most 5 points from the full binary projective geometry $\mathrm{PG}(3,2)$ of rank 4 (or dimension 3 ).

(7.4.1) If $G^{c}$ contains an affine plane, then $G$ splits.

Proof. In PG(3,2), let $\pi$ be the projective plane obtained by taking the linear span of the affine plane contained in $G^{c}$. Let $m$ be the line $G \cap \pi$, and let $\pi^{\prime}$ and $\pi^{\prime \prime}$ be the two other projective planes containing the line $m$. Then $G \subseteq \pi^{\prime} \cap \pi^{\prime \prime}$, and $G$ is the union of the two flats $G \cap \pi^{\prime}$ and $G \cap \pi^{\prime \prime}$.

Before proceeding to the case analyses, we note that the automorphism group of $\operatorname{PG}(3,2)$ is transitive on bases; hence, we may choose coordinates for a basis in any way we wish, subject only to the restriction of linear independence.

We first consider the case when $G^{c}$ has exactly five points. If $G^{c}$ has rank 3 , then it contains an affine plane. Thus, as $G$ does not split, we can assume that $G^{c}$ has rank 4. There are two possibilities.

(7.4.2) $G^{c}$ contains a three-point line. As $G^{c}$ has rank 4 , this line is the only three point line in $G^{c}$. Choose as coordinates for the points in the line $(0,1,1,0),(1,0,1,1)$, and $(1,1,0,1)$. There are two remaining points in $G^{c}$, and we may choose them to be $(1,1,1,1)$ and $(0,1,1,1)$. From this, we see that $G$ is isomorphic to $C_{10}$.

As $G^{c}$ contains no affine plane, it cannot contain a circuit of size 4 . Hence, we have one more case.

(7.4.3) $G^{c}$ contains no circuits of size 3 or 4 . We may choose $(1,1,1,0),(1,1,0,1)$, $(1,0,1,1)$, and $(0,1,1,1)$, the four vectors with exactly three nonzero coordinates, to be four of the points in $G^{c}$. As the fifth point does not form a circuit of size 3 or 4 with these four points, it can only be the vector $(1,1,1,1)$. Thus, $G$ is isomorphic to $M\left(K_{5}\right)$.

When $G^{c}$ has three or four points, there are again two possibilities: $G^{c}$ contains a three-point line, in which case $G$ contains $C_{10}$, or, $G^{c}$ is independent, in which case $G$ contains $M\left(K_{5}\right)$. The proofs are similar to those in (7.4.2) and (7.4.3). When $G^{c}$ has 0,1 , or 2 points, similar analyses show that $G$ contains both $C_{10}$ and $M\left(K_{5}\right)$.

This completes the proof of (7.1).

For later use, we describe here in greater detail the geometries with 12 points occurring in the previous proof.

(7.5) The binary geometries $C_{12}$ and $D_{12}$ are the two nonisomorphic binary geometries of rank 4 with 12 points. The geometry $C_{12}$ is obtained by removing from PG $(3,2)$ a three-point line. This geometry does not contain $M\left(K_{5}\right)$ as a minor. To see this, observe that $C_{12}$, having the same rank as $M\left(K_{5}\right)$, must contain $M\left(K_{5}\right)$ as 
a subgeometry. However, $C_{12}$ has critical exponent 2 (by construction), while $M\left(K_{5}\right)$ has critical exponent 3 . The geometry $D_{12}$ is obtained by removing from PG $(3,2)$ an independent set of size 3 . $D_{12}$ contains $M\left(K_{5}\right)$ as a subgeometry.

Another result we shall need is the following.

(7.6) LEMMA. Let $\Gamma(x, G)$ be a bond graph such that

(a) $\Gamma(x, G)$ contains a topological $K_{4}$, and

(b) the base vertices of the topological $K_{4}$ form a spanning set of the geometry $G$.

Then $G$ contains $M\left(K_{5}\right)$ or $F_{7}$ as a minor.

Proof. If the base vertices of the topological $K_{4}$ form an independent set, then, by (7.1), $G$ contains $M\left(K_{5}\right)$ or $C_{10}$. But $C_{10}$ contains $F_{7}$ as a contraction. If the base vertices are dependent, then they form an affine plane by (4.1). Hence, by (b), there are no other vertices. It is now easy to check that $G$ is isomorphic to $F_{7}$.

8. Proofs. We are finally ready to give the proofs of (2.5) and (2.6). These proofs are very similar, and only the more intricate proof of (2.6) will be given in detail.

To prove (2.6), we first show that the maximun. growth rate of $\mathscr{E} x\left(M\left(K_{5}\right), C_{10}\right)$ is at most 4.

(8.1) THEOREM. Let $\mathscr{C}$ be a minor-closed class of binary geometries with maximum growth rate at least 5 . Then $\mathscr{C}$ contains $M\left(K_{5}\right)$ or $C_{10}$.

Proof. A subgraph of a bond graph $\Gamma(x, G)$ is said to be an independent topological $K_{4}$ if it is a topological $K_{4}$ whose base vertices form an independent set in $G$.

(8.2) Lemma. There exists a bond graph $\Gamma(x, G)$ with $G$ in $\mathscr{C}$ containing an independent topological $K_{4}$.

Proof. Let $G$ be a maximum size geometry in $\mathscr{C}$ of rank $n_{0}$, where $g\left(n_{0}\right) \geqslant 5$. Let $x$ be any copoint of $G$. By (3.6), every vertex in the bond graph $\Gamma(x, G)$ has degree at least 4 . Hence, by Pelikán's theorem $(6.2), \Gamma(x, G)$ contains a topological $K_{5}^{-}$. Let $a, b, c, d$ and $a, b, c, e$ be the base vertices of the two topological $K_{4}$ 's contained in the topological $K_{5}^{-}$. By (1.3), at least one of the sets $\{a, b, c, d\}$ or $\{a, b, c, e\}$ is independent in $G$. Hence, $\Gamma(x, G)$ contains an independent topological $K_{4}$.

Among all the bond graphs constructed from geometries in $\mathscr{C}$ containing independent topological $K_{4}$ 's, choose one, $\Gamma(x, G)$, which is minimal in the following sense:

A. Every point in $G$ occurs as a vertex or edge label in $\Gamma(x, G)$.

B. The rank of $G$ is as small as possible.

C. Subject to $B$, the number of vertices is as small as possible.

D. Subject to $B$ and $C$, the number of edges is as small as possible.

Let $\Delta$ be an independent topological $K_{4}$ in $\Gamma(x, G)$ with base vertices $a, b, c, d$. By Condition $\mathrm{C}$ and $(4.3), \Gamma(x, G)$ consists of $\Delta$ and possibly some extra edges. Let us call the edges in $\Delta$ transverse edges, and the extra edges crossing edges. 
As long as at least one endpoint of a transverse edge is not a base vertex, the edge can be contracted graphically, and $\Delta$ would remain a topological $K_{4}$ with base vertices $a, b, c, d$. Unfortunately, this is not true of crossing edges. The rest of our proof is devoted to considering crossing edges. In all the case analyses to follow, our aim is to prove

(8.3) Lemma. Every edge label in $\Gamma(x, G)$ is in the closure of the set $\{a, b, c, d\}$ of base vertices.

Once (8.3) is proved, the proof of (8.1) can be completed in the following way. Since every vertex in $\Gamma(x, G)$ is joined by a path to a base vertex, (8.3) and (4.1)(b) imply that every vertex of $\Gamma(x, G)$ is in the closure of $\{a, b, c, d\}$. Hence, by Condition A, $G$ has rank 4. From (7.1), we conclude that $G$ contains $M\left(K_{5}\right)$ or $C_{10}$, thus proving (8.1).

We now proceed to the case analyses. We begin with the (easiest) case when

(A) there are no crossing edges.

Suppose there exists an edge label $\alpha$ in $\Gamma(x, G)$ not in the closure of $\{a, b, c, d\}$. Then $\alpha$ cannot be the label of an edge $\{u, v\}$, where $u$ and $v$ are both base vertices. Thus, in $\Gamma(x, G) / / \alpha$, the graph obtained from $\Gamma(x, G)$ by contracting all the edges labelled by $\alpha$, the vertices $a, b, c, d$ are still the base vertices of a topological $K_{4}$. In addition, $\{a, b, c, d\}$ remains independent in $G / \alpha$. Hence, by (4.5), $\Gamma(x / \alpha, G / \alpha)$ contains an independent topological $K_{4}$, contradicting minimality. We conclude that (8.3) is true in this case.

Now suppose that $\{u, v\}$ is a crossing edge. By minimality, $u$ and $v$ are on different paths between base vertices in $\Delta$. There are three possibilities:

I. $u$ is a base vertex and $v$ is a nonbase vertex on a path between base vertices not containing $u$.

II. $u$ and $v$ are not base vertices and are on two paths between base vertices sharing a common base vertex.

III. $u$ and $v$ are not base vertices and are on two disjoint paths between base vertices.

We shall first consider the case when

(B) there is a crossing edge of type I.

By relabelling, we may assume that the crossing edge is of the form $\{b, u\}$ where $u$ is a vertex on the path from $c$ to $d$ (see Figure 1). Our first step is to show that certain paths in $\Delta$ consist of single edges.

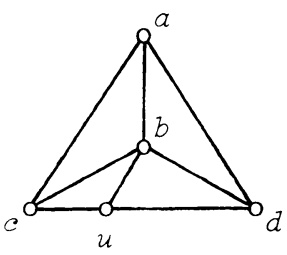

type I

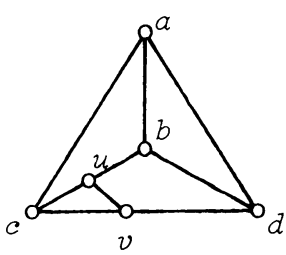

type II

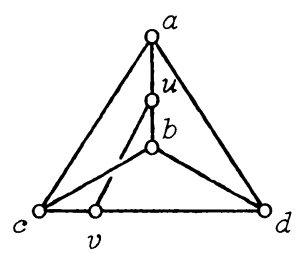

type III

Figure 1. Crossing edges 
(8.4) LEMMA. $\{a, b\}$ is an edge.

Proof. We first observe that $\{b, c, d, u\}$ is independent. To see this, suppose it is dependent. Then, as $\{b, u\}$ is an edge, $\{c, d\}$ is also an edge by (4.2). Thus, if the vertex $u$ is deleted, $\Gamma(x, G)$ would still contain an independent topological $K_{4}$, contradicting minimality.

Now, $b, c, d, u$ are the base vertices of an independent topological $K_{4}$ not using any of the edges in the path from $a$ to $b$. Comparing the number of edges in this $K_{4}$ and in $\Delta$, we conclude that $\{a, b\}$ must be an edge by Condition $\mathrm{D}$.

We shall use the argument in the proof of (8.4) repeatedly and refer to it as the single edge argument.

(8.5) Lemma. $\{a, b, c, u\}$ and $\{a, b, d, u\}$ are both independent, and $\{b, c\}$ and $\{b, d\}$ are edges.

Proof. By (1.3), at least one of $\{a, b, c, u\}$ and $\{a, b, d, u\}$ is independent. By symmetry, we may assume that $\{a, b, d, u\}$ is independent. By the single edge argument, $\{b, c\}$ is an edge.

Now suppose that $\{a, b, c, u\}$ is dependent. As $\{b, c\}$ is an edge, $\{a, u\}$ is also an edge by (4.2). But then $\{a, b, d, u\}$ are the base vertices of an independent topological $K_{4}$ not using the vertex $c$, contradicting minimality. Hence, $\{a, b, c, u\}$ is independent, and by the single edge argument $\{b, d\}$ is an edge.

Our second step is to show that all the other crossing edges in $\Gamma(x, G)$ are of the form $\{b, v\}$ for some vertex $v$.

(8.6) LEMMA. There are no crossing edges of types II or III.

Proof. As $\{a, b\},\{b, c\}$, and $\{b, d\}$ are edges, there cannot be any crossing edges of type III.

Now suppose there is a crossing edge $\{s, t\}$ of type II with $s$ on the path from $a$ to $c$, and $t$ on the path from $a$ to $d$. Then, by detouring through $\{s, t\}$, we obtain an independent topological $K_{4}$ on the base vertices $b, c, d, u$ not using the vertex $a$, contradicting minimality. The other cases can be ruled out because $\{a, b\},\{b, c\}$, and $\{b, d\}$ are edges or are handled in the same manner.

(8.7) Lemma. A crossing edge of type I must be of the form $\{b, v\}$.

Proof. If $\{c, v\}$ is a crossing edge, then, by the arguments in (8.4) and (8.5) applied to $c$ instead of $b,\{c, d\}$ is an edge, contradicting minimality. In a similar way, crossing edges of the form $\{d, v\}$ are ruled out. Now suppose $\{a, v\}$ is a crossing edge with $v$ on the path from $c$ to $u$. By detouring through $v$, we obtain an independent topological $K_{4}$ on the base vertices $a, b, d, u$ not using the vertex $c$, contradicting minimality. A similar argument works if $v$ is on the path from $u$ to $d$.

Our next result shows that crossing edges of the form $\{b, v\}$ are distributed in a "nice" way in $\Gamma(x, G)$. 
(8.8) LEMmA. Let $c=u_{0}, u_{1}, u_{2}, \ldots, u_{m}=d$ be the sequence of vertices in the path from c to $d$. Then $m$ is even, $\left\{b, u_{2 i}\right\}$ is an edge for every $i$, and

$$
l\left(u_{2 i-2}, u_{2 i-1}\right)=l\left(b, u_{2 i}\right)=l\left(u_{2 i+1}, u_{2 i+2}\right)
$$

whenever the subscripts are between 0 and $m$.

The proof of (8.8) is facilitated by the following lemma.

(8.9) Lemma. Let $0 \leqslant i<m$. Suppose that $\left\{b, u_{i}\right\}$ is an edge. Then $i \leqslant m-2$ and $\left\{b, u_{i+2}\right\}$ is an edge. Further, $l\left(u_{i}, u_{i+1}\right)=l\left(b, u_{i+2}\right)$ and $l\left(b, u_{i}\right)=l\left(u_{i+1}, u_{i+2}\right)$.

Proof. Let $\alpha$ be the label of the edge $\left\{u_{i}, u_{i+1}\right\}$.

(8.9.1) If $\{a, c\}$ is an edge, then $\alpha \neq l(a, c)$.

Proof. Let $\beta=l\left(b, u_{i}\right)$. Then as $\alpha$ and $\beta$ label incident edges, $\alpha \neq \beta$ by (4.1)(d). Now, if $\{a, d\}$ is an edge and $\beta=l(a, d)$, then $a, b, d, u_{i}$ are coplanar. Since $\{b, d\}$ is an edge, $\left\{a, u_{i}\right\}$ is an edge by (4.2), contrary to (8.7). Moreover, as every crossing edge is incident on $b, \beta$ is not the label of any other crossing edge. Hence, $\Gamma(x, G) / / \beta$ is as shown in Figure 2 .

By (4.5), $\Gamma(x / \beta, G / \beta)$ contains $\Gamma(x, G) / / \beta$ as an edge subgraph. Since $l(a, c)=$ $l\left(b, u_{i+1}\right)$ in $\Gamma(x / \beta, G / \beta), a, b, c, u_{i+1}$ are coplanar in $G / \beta$. Hence, $\left\{a, b, c, u_{i+1}\right\}$ is independent by (1.3). Further, as $\{a, b\}$ is an edge, $\left\{c, u_{i+1}\right\}$ is also an edge by (4.2), and there exists a path from $u_{i+1}$ to $a$ (see Figure 2). Thus, $a, b, d, u_{i+1}$ are the base vertices of an independent topological $K_{4}$ in $\Gamma(x / \beta, G / \beta)$. This contradicts the minimality of $\Gamma(x, G)$.

(8.9.2) If $\{a, d\}$ is an edge, then $\alpha \neq l(a, d)$.

The proof is similar to that of (8.9.1), except that we contract by $l(b, d)$.

(8.9.3) There is a crossing edge $\{b, v\}$ labelled by $\alpha$.

Proof. Suppose not. Then by (8.9.1) and (8.9.2), $\alpha$ can only be the label of transverse edges with at most one endpoint a base vertex. In $\Gamma(x, G) / / \alpha$, and hence in $\Gamma(x / \alpha, G / \alpha), a, b, c, d$ are still the base vertices of a topological $K_{4}$ with $\left\{b, u_{i}\right\}$ a crossing edge. By (1.3), at least one of $\left\{a, b, c, u_{i}\right\}$ or $\left\{a, b, d, u_{i}\right\}$ is independent in $G / \alpha$, and it forms the base vertices of an independent topological $K_{4}$, contrary to the minimality of $\Gamma(x, G)$.

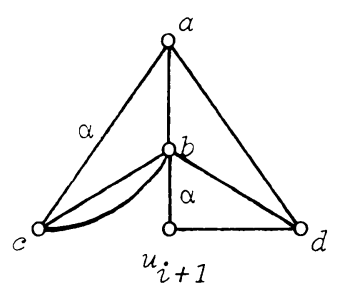

$\Gamma(x, G) / / B$

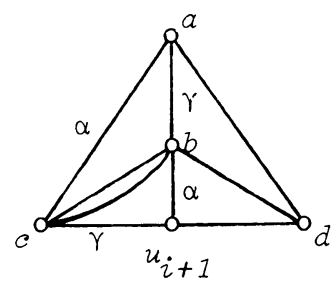

$\Gamma(x / B, G / B)$ 
Consider the crossing edge $\{b, v\}$ labelled by $\alpha$. As $l(b, v)=l\left(u_{i}, u_{i+1}\right), b, u_{i}$, $u_{i+1}, v$ are coplanar. Since $\left\{b, u_{i}\right\}$ is an edge, $\left\{u_{i+1}, v\right\}$ is also an edge by (4.2). As there are no crossing edges of types II or III and $\Gamma(x, G)$ is minimal, $v$ must equal $u_{i+2}$. Hence, $\left\{b, u_{i+2}\right\}$ is an edge labelled by $\alpha$. Finally, by $(4.2), l\left(b, u_{i}\right)=$ $l\left(u_{i+1}, u_{i+2}\right)$. This completes the proof of (8.9).

Now $\{b, c\}$ is an edge and so, by (8.9), $\left\{b, u_{2}\right\}$ is also an edge. Continuing this argument, we obtain (8.8).

Using the argument in the proof of (8.8), we also obtain

(8.10) LeMma. The paths from a to $c$ and a to d either consist of a single edge or are of the form described in (8.8).

Let $a=v_{0}, v_{1}, v_{2}, \ldots, v_{m}=c$ be the sequence of vertices in the path from $a$ to $c$. Define $c^{\prime}$ to be $c$ if $m=1$ and $u_{2}$ if $m \geqslant 2$. Define $d^{\prime}$ similarly. The set $\left\{a, b, c^{\prime}, d^{\prime}\right\}$ is independent (otherwise, $\left\{c^{\prime}, d^{\prime}\right\}$ is an edge by (4.2), and $b, c, c^{\prime}, d$ are the base vertices of an independent topological $K_{4}$ not using the vertex $a$, contradicting minimality) and forms the base vertices of an independent topological $K_{4}$. Exchanging the labels $c, c^{\prime}$ and $d, d^{\prime}$ if necessary, we may assume that in $\Delta$ the independent topological $K_{4}$ 's contained in $\Gamma(x, G),\{a, b\},\{b, c\},\{b, d\}$ are edges, the paths from $a$ to $c$ and $a$ to $d$ each contain at most two edges, and the path from $c$ to $d$ is of the form described in (8.8). Let $\Delta^{-}$be the subgraph of $\Gamma(x, G)$ obtained by removing all the vertices $u_{1}, u_{2}, \ldots, u_{m-1}$ inside the path from $c$ to $d$. By (8.10), every vertex and edge label in $\Delta^{-}$is in the closure $\overline{\{a, b, c, d\}}$ of $\{a, b, c, d\}$ in $G$.

Our final step is to show that every edge label in the path from $c$ to $d$ is in $\overline{\{a, b, c, d\}}$. Suppose that there exists an edge in the path labelled by a point not in $\{a, b, c, d\}$. Going along the path from $c$ to $d$, let $\left\{u_{k}, u_{k+1}\right\}$ be the first such edge, and let $\gamma=l\left(u_{k}, u_{k+1}\right)$. By $(*)$ in (8.8), $k$ is even. Further, $u_{k} \in \overline{\{a, b, c, d\}}$, but $u_{k+1} \notin \overline{\{a, b, c, d\}}$.

Let $w$ be the set of edge labels in $\overline{\{a, b, c, d\}}$. Since $w=x \wedge \overline{\{a, b, c, d\}}, w$ is a closed set and $\gamma \notin w$. Let $m$ be a modular complement to $\gamma \vee w$ in the interval $[w, x]$ in the lattice of flats of $G$ (i.e. $m$ is a closed set in $[w, x]$ such that $m \wedge(\gamma \vee w)=w, m \vee(\gamma \vee w)=x$, and $m$ and $\gamma \vee w$ form a modular pair). By computing rank, $m$ is a coline contained in $x$.

What are the other copoints above $m$ ? One of them is $z=m \vee a$.

(8.11) LemMA. $\overline{\{a, b, c, d\}}$ is contained in $z$.

Proof. It suffices to show that the points $b, c$, and $d$ are in $z$. But $b, c$, and $d$ are connected to $a$ by paths in the subgraph $\Delta^{-}$. Since every edge label in $\Delta^{-}$is in $w$ and $w \subseteq z, b, c, d \in z$ by (4.1)(b).

(8.12) Lemma. The point $u_{k+1}$ is not contained in $x$ or $z$.

Proof. As $u_{k+1}$ is a vertex, it is certainly not in $x$. Suppose $u_{k+1} \in z$. Since $u_{k}$ $\in \overline{\{a, b, c, d\}}$, it is in $z$. Hence, $\gamma=l\left(u_{k}, u_{k+1}\right)$ is in $z$. Since $\gamma$ is an edge label, $\gamma \in x$, and so, $\gamma \in x \wedge z=m$, contradicting our choice of $m$.

From (8.12), we conclude that there is a third copoint $y=m \vee u_{k+1}$ above $m$. As $G$ is binary, $x, y$, and $z$ are all the copoints above $m$. 
To finish Case $\mathrm{B}$, we shall prove

(8.13) Proposition. The bond graph $\Gamma(y, G)$ contains an independent topological $K_{4}$ but has strictly fewer vertices than $\Gamma(x, G)$.

Proof. Consider the bond graph $\Gamma(y, G)$. As the set $z \backslash m$ of points in $z$ but not in $m$ is contained in both $x^{c}$ and $y^{c}$, the vertices in $z \backslash m$ are common to both $\Gamma(x, G)$ and $\Gamma(y, G)$. Further, if $s$ and $t$ are in $z \backslash m$, then the third point on the line $s \vee t$ (if it is in $G$ ) is in $m$. We have thus proved

(8.13.1) The induced subgraphs $\Gamma(x, G) \mid z \backslash m$ and $\Gamma(y, G) \mid z \backslash m$ are identical as labelled graphs. In particular, $\Gamma(y, G)$ contains $\Delta^{-}$as a subgraph.

Next, we show that there is a path from $c$ to $d$ in $\Gamma(y, G)$ containing no vertices from $\Delta^{-}$other than $c$ and $d$. Recall that the path from $c$ to $d$ in $\Gamma(x, G)$ is of the form $c=u_{0}, u_{1}, \ldots, u_{m}=d$ with $\left\{b, u_{2 i}\right\}$ an edge for $0 \leqslant 2 i \leqslant m$. Let $\alpha_{2 i}=$ $l\left(b, u_{2 i}\right)$. In the sequence $u_{0}, u_{1}, \ldots, u_{m}$ of vertices, let $u_{2 j(0)}, u_{2 j(1)}, \ldots$, be the subsequence of vertices which has an even subscript and is in $z$. Note that $u_{2 j(0)}=c$, the last vertex in the subsequence is $u_{m}=d$, and the vertices in this subsequence, being in $z$, are vertices in both $\Gamma(x, G)$ and $\Gamma(y, G)$.

(8.13.2) If $j(p+1)=j(p)+1$, then $u_{2 j(p)}$ and $u_{2 j(p+1)}$ are connected by the path $u_{2 j(p)}, u_{2 j(p)+1}, u_{2 j(p)+2}=u_{2 j(p+1)}$ in $\Gamma(y, G)$.

ProOF. We first check that $u_{2 j(p)+1}$ is in $z$ and is indeed a vertex in $\Gamma(y, G)$. To do this, observe that since $b, u_{2 j(p)+2} \in z, l\left(b, u_{2 j(p)+2}\right) \in m$. Since $l\left(b, u_{2 j(p)+2}\right)$ $=l\left(u_{2 j(p)}, u_{2 j(p)+1}\right)$ (by (8.8)) and $u_{2 j(p)} \in z, u_{2 j(p)+1}$ is also in $z$. It remains to observe that, by $(8.13 .1),\left\{u_{2 j(p)}, u_{2 j(p)+1}\right\}$ and $\left\{u_{2 j(p)+1}, u_{2 j(p)+2}\right\}$ are edges in both $\Gamma(x, G)$ and $\Gamma(y, G)$.

(8.13.3) If $j(p+1)>j(p)+1$, then $u_{2 j(p)}$ and $u_{2 j(p+1)}$ are connected by the path

$$
\begin{array}{r}
u_{2 j(p)}, \alpha_{2 j(p)+2}, u_{2 j(p)+3}, \alpha_{2 j(p)+4}, u_{2 j(p)+5}, \ldots, \\
\alpha_{2 j(p+1)-4}, u_{2 j(p+1)-3}, \alpha_{2 j(p+1)+2}, u_{2 j(p+1)} .
\end{array}
$$

The "new" path in $\Gamma(y, G)$ is obtained from the "old" path

$$
u_{2 j(p)}, u_{2 j(p)+1}, u_{2 j(p)+2}, u_{2 j(p)+3}, \ldots, u_{2 j(p+1)-2}, u_{2 j(p+1)-1}, u_{2 j(p+1)}
$$

in $\Gamma(x, G)$ by removing the two extreme odd subscripted vertices $u_{2 j(p)+1}$ and $u_{2 j(p+1)-1}$, and replacing the "interior" even subscripted vertices $u_{2 j(p)+2}$, $u_{2 j(p)+4}, \ldots, u_{2 j(p+1)-2}$ by $\alpha_{2 j(p)+2}, \alpha_{2 j(p)+4}, \ldots, \alpha_{2 j(p+1)-2}$ (see Figure 3).
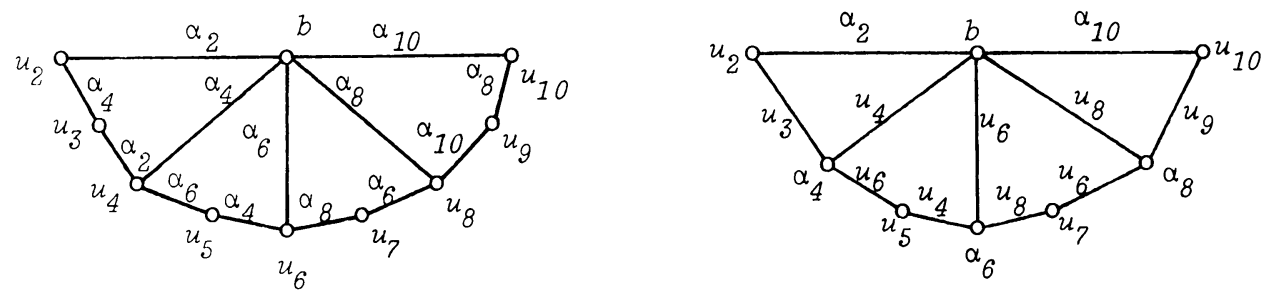

FigurF 3. A path and its transformation. Here, $j(p)=1$ and $j(p+1)=5$. 
Proof. Observe first that the "interior" even subscripted vertices $u_{2 j(p)+2}$, $u_{2 j(p)+4}, \ldots, u_{2 j(p)-2}$, in the "old" path in $\Gamma(x, G)$ are not in $z$. As they are vertices in $\Gamma(x, G)$, they are not in $x$. Hence, they must be in $y \backslash m$. From this, we deduce three easy consequences.

(8.13.4) The two extreme odd subscripted vertices $u_{2 j(p)+1}$ and $u_{2 j(p+1)-1}$ are in $y \backslash m$.

Proof. It suffices to show that they are not in $z$. If $u_{2 j(p)+1} \in z$, then $l\left(u_{2 j(p)}, u_{2 j(p)+1}\right) \in m$. By $(*)$ in (8.8), $l\left(b, u_{2 j(p)+2}\right)$ is in $m$, and so $u_{2 j(p)+2}$ is in $z$, contradicting our assumption that $j(p+1)>j(p)+1$. The assertion for $u_{2 j(p+1)-1}$ follows similarly.

(8.13.5) The edge labels $\alpha_{2 j(p)+2}, \alpha_{2 j(p)+4}, \ldots, \alpha_{2 j(p+1)-2}$ in $\Gamma(x, G)$ are in $x \backslash m$.

Proof. It suffices to show that they are not in $m$. Since $b \in z \backslash m$ and $u_{2 j(p)+2} \in$ $y \backslash m$, the modular line $b \vee u_{2 j(p)+2}$ is not in $m$ and, hence, must intersect the copoint $x$ at a point not in $m$. Thus, the third point, $\alpha_{2 j(p)+2}$, is in $x \backslash m$. A similar argument works for the other edge labels.

(8.13.6) The odd subscripted vertices $u_{2 j(p)+3}, u_{2 j(p)+5}, \ldots, u_{2 j(p+1)-3}$ are in $z \backslash m$.

Proof. Since $u_{2 j(p)+2} \in y \backslash m$ and $l\left(u_{2 j(p)+2}, u_{2 j(p)+3}\right)=\alpha_{2 j(p)+4} \in x \backslash m$, $u_{2 j(p)+3} \in z \backslash m$, by the same argument as in (8.13.5). The assertion for the other vertices follows similarly.

By (8.13.5) and (8.13.6), the vertices in the path $(\dagger)$ are indeed vertices in $\Gamma(y, G)$. It remains to show that consecutive vertices in $(\dagger)$ are joined by an edge in $\Gamma(y, G)$. But, it is easy to check that

$$
\begin{aligned}
& \left\{u_{2 j(p)}, \alpha_{2 j(p)+2}\right\} \text { is an edge labelled by } u_{2 j(p)+1}, \\
& \left\{\alpha_{2 j(p)+2}, u_{2 j(p)+3}\right\} \text { is an edge labelled by } u_{2 j(p)+4}, \\
& \left\{u_{2 j(p)+3}, \alpha_{2 j(p)+4}\right\} \text { is an edge labelled by } u_{2 j(p)+2}, \\
& \quad \vdots \\
& \left\{\alpha_{2 j(p+1)-2}, u_{2 j(p+1)}\right\} \text { is an edge labelled by } u_{2 j(p+1)-1}
\end{aligned}
$$

(see Figure 3). This completes the proof of (8.13.3).

By concatenating the paths in (8.13.2) and (8.13.3), we obtain a path from $c$ to $d$ in $\Gamma(y, G)$ not containing any vertices in $\Delta^{-}$other than $c$ and $d$. We conclude that $a, b, c, d$ are the base vertices of a topological $K_{4}$ in $\Gamma(y, G)$.

Now, by $(8.8), l\left(b, u_{k+2}\right)=l\left(u_{k}, u_{k+1}\right)=\gamma$, and so $l\left(b, u_{k+2}\right) \in x \backslash m$. As $b \in$ $z \backslash m$, this implies $u_{k+2} \in y \backslash m$. Thus, there exists an even subscripted vertex not in the subsequence $u_{2 j(1)}, u_{2 j(2)}, \ldots$ Hence, the hypothesis of (8.13.3) holds for some $j(p)$. Since the "new" path $(\dagger)$ in $\Gamma(y, G)$ given in (8.13.3) has two fewer vertices than the "old" path in $\Gamma(x, G)$, we conclude that the number of vertices in $\Gamma(y, G)$ is strictly less than the number of vertices in $\Gamma(x, G)$. This completes the proof of (8.13).

Since (8.13) contradicts the minimality of $\Gamma(x, G)$, every vertex in the path from $c$ to $d$ in $\Gamma(x, G)$ is in $\overline{\{a, b, c, d\}}$. Thus, (8.3) holds in Case (B).

Next, we consider the case when

(C) there is a crossing edge $\{u, v\}$ of type II but no crossing edges of type I. 
By relabelling, we may assume that $u$ is a vertex on the path from $c$ to $b$, and $v$ a vertex on the path from $c$ to $d$ (see Figure 1). We shall first show that certain paths in $\Delta$ are in fact edges.

(8.14) Lemma. $\{a, b\},\{a, d\},\{c, u\},\{c, v\}$, and $\{b, d\}$ are edges.

Proof. As in the proof of (8.4), $\{c, d, u, v\}$ is independent. By the single edge argument, $\{a, b\}$ is an edge. Similarly, $\{a, d\}$ is an edge.

Next, observe that $\{a, b, d, v\}$ is independent. (Otherwise, as $\{a, d\}$ is an edge, $\{b, v\}$ is also an edge by (4.2). But $\{b, v\}$ is a crossing edge of type I.) By the single edge argument, $\{c, u\}$ is an edge. Similarly, $\{c, v\}$ is an edge.

Finally, since $\{c, v\}$ is an edge but $\{a, v\}$, being of Type $\mathrm{I}$, is not an edge, $\{a, c, u, v\}$ is independent. By the single edge argument, $\{b, d\}$ is an edge.

From (8.14), it is easy to check that there cannot be any crossing edges of type III.

(8.15) Lemma. Besides $\{u, v\}$, there are no other crossing edges of type II.

Proof. Let $\{s, t\}$ be a crossing edge of type II. By (8.14), $s$ and $t$ are on paths from $c$ to $a, b$, or $d$. Using the argument in (8.14), $\{c, s\}$ and $\{c, t\}$ are edges. Hence, by minimality, $\{s, t\}$ either equals $\{u, v\}$ or shares one endpoint with $\{u, v\}$. By symmetry, we may assume that $\{s, t\}=\{u, t\}$, where $t$ is a vertex on the path from $c$ to $a$.

Now suppose $\{c, t, u, v\}$ is independent. Then it forms the base vertices of an independent topological $K_{4}$ not using the vertex $b$, contradicting minimality. On the other hand, if $\{c, t, u, v\}$ is dependent, then $\{t, v\}$ is an edge by (4.2). Further, $\{a, t, u, v\}$ is independent and forms the base vertices of a topological $K_{4}$ not using the vertex $c$, contradicting minimality again.

(8.16) Lemma. The edge label $\alpha$ of $\{u, v\}$ is in $\overline{\{a, b, c, d\}}$.

Proof. Let $\beta=l(c, u)$. Then by (4.1)(d), $\beta \neq \alpha$. If $\beta$ is not in $\overline{\{a, b, c, d\}}$, then, using the argument in Case (A), $a, b, c, d$ are the base vertices of an independent topological $K_{4}$ in the bond graph $\Gamma(x / \beta, G / \beta)$, contradicting minimality. Thus, $\beta$, and hence $u$, is in $\overline{\{a, b, c, d\}}$. Similarly, $v \in \overline{\{a, b, c, d\}}$. As $u, v, \alpha$ are collinear,

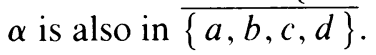

Finally, suppose $\gamma$ is an edge label in $\Gamma(x, G)$ not equal to $\alpha$. Since $\gamma$ does not label a crossing edge, $\gamma$ must be in $\overline{\{a, b, c, d\}}$ by the same argument as in Case (A). We have thus proved (8.3) for Case (C).

Our final case is when

(D) there is a crossing edge of type III but no crossing edges of types I or II.

There are three pairs of paths between base vertices in $\Delta$ not sharing a common base vertex. We first dispose of the case when

(D1) there are two crossing edges of type III and they are incident on different pairs of paths in $\Delta$. 


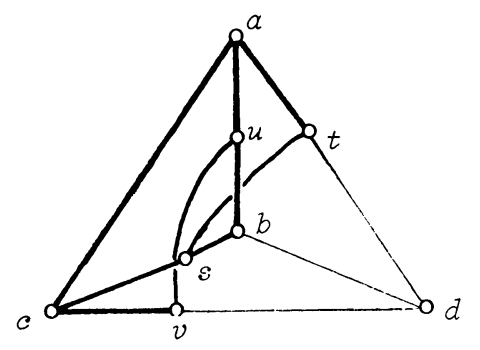

(a)

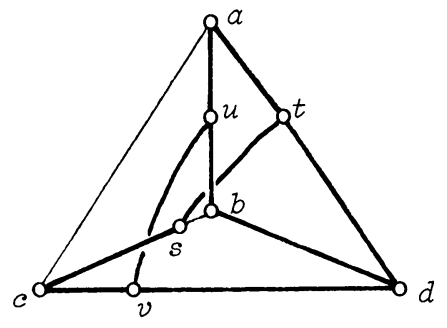

(b)

Figure 4

Let these edges be $\{u, v\}$ and $\{s, t\}$ and suppose that they are as shown in Figure 4a.

(8.17) LEMMA. The following sets of points are coplanar: $\{a, c, s, u\},\{a, c, t, v\}$, and $\{s, t, u, v\}$.

Proof. It suffices to show that these sets are dependent. If $\{a, c, s, u\}$ is independent, then it forms the base vertices of an independent topological $K_{4}$ not using the vertex $d$. This topological $K_{4}$ is shown by the thicker lines in Figure 4a. As $\Gamma(x, G)$ is minimal, $\{a, c, s, u\}$ must be dependent. Similarly, $\{a, c, t, v\}$ is also dependent. Finally, $\{s, t, u, v\}$ is dependent because it is the symmetric difference of two circuits in a binary geometry.

(8.18) LEMMA. $\{a, c\}$ is an edge.

Proof. Since $u, v, s, t$ are coplanar, $\{d, u, v, t\}$ is an independent set. In addition, $d, u, t, v$ are the base vertices of a topological $K_{4}$ not using any of the edges in the paths from $a$ to $c$ and $b$ to $s$. This topological $K_{4}$ is shown by the thicker lines in Figure $4 b$. Thus, by the single edge argument, $\{a, c\}$ and $\{b, s\}$ are both edges.

To finish Case (D1), observe that since $\{a, c, s, u\}$ is coplanar and $\{a, c\}$ is an edge, $\{s, u\}$ is also an edge by (4.2). But $\{s, u\}$ is a crossing edge of Type II, contradicting our assumption. Thus, Case (D1) is impossible.

There remains the case when

(D2) all the crossing edges of type III are incident on the same pair of paths between base vertices.

We may suppose that the pair of paths are the paths from $a$ to $b$ and $c$ to $d$.

(8.19) LEMMA. If $\alpha$ is the label of a crossing edge, then $\alpha \in \overline{\{a, b, c, d\}}$.

Proof. Suppose not. On the path from $a$ to $b$, let $u$ be the first vertex incident on a crossing edge $\{u, v\}$ having a label $\alpha$ not in $\overline{\{a, b, c, d\}}$, and let $s$ be the last vertex incident on a crossing edge $\{s, t\}$ (see Figure 5a for an example). Consider the bond graph $\Gamma(x / \alpha, G / \alpha)$. By (4.6), it contains one of the subgraphs shown in Figure 5b, c. 


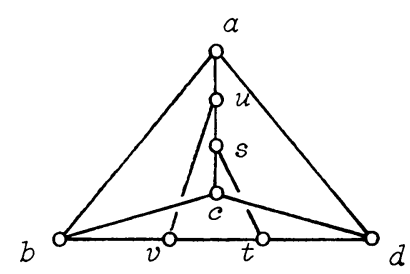

(a)

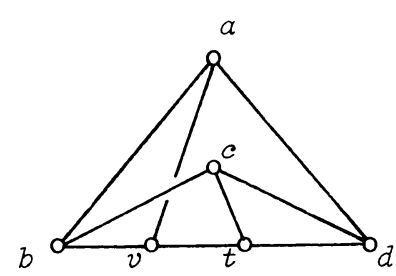

(b)

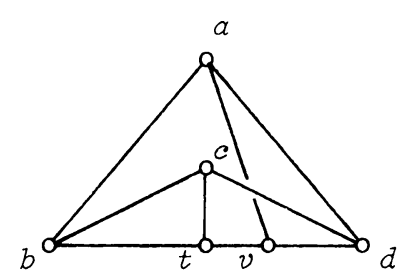

(c)

Figure 5. Contracting crossing edges of type III. (Not all the edges are shown.)

If $\{b, c, t, d\}$ is independent, then $\Gamma(x / \alpha, G / \alpha)$ contains an independent topological $K_{4}$, contradicting minimality. If $\{b, c, t, d\}$ is dependent, then $\{a, c, t, d\}$ and $\{a, c, t, b\}$ are both independent, and depending on the position of $v$ relative to $t$, at least one of them forms the base vertices of an independent topological $K_{4}$, contradicting minimality.

We can now use the argument in Case (A) to conclude that every edge label in $\Gamma(x, G)$ is in $\overline{\{a, b, c, d\}}$, thus proving (8.3) for Case (D2).

This completes our case analyses and our proof of (8.1).

We shall now exhibit a subclass of geometries in $\mathscr{E} x\left(M\left(K_{5}\right), C_{10}\right)$ attaining the maximum growth rate of 4 .

(8.20) Parallel connections of Fano planes. We define the class $\mathcal{O}\left[F_{7}\right]$ of geometries recursively. Let $\mathcal{O}_{1}=\left\{F_{7}\right\}$, and let $\mathscr{O}_{n}$ be the class of geometries obtained by taking a geometry $H$ in $\mathcal{O}_{n-1}$ and a copy of $F_{7}$, choosing a three-point line in each, and constructing the (generalized) parallel connection of $H$ and $F_{7}$ along that three-point line. (For details on parallel connections, see $\left[2\right.$, p. 19].) The class $\mathcal{O}\left[F_{7}\right]$ is defined by

$$
\mathcal{O}\left[F_{7}\right]=\bigcup_{n=1}^{\infty} \mathcal{O}_{n}
$$

It is easy to check that

(1) If $H \in \mathcal{O}_{n}$, then $H$ has rank $n+2$ and size $4 n+3$.

(2) If $H \in \mathcal{O}\left[F_{7}\right]$ and $K$ is a minor of rank at least 4 in $H$, then $K$ splits.

By (2), a geometry in $\mathcal{O}\left[F_{7}\right]$ does not contain $M\left(K_{5}\right)$ or $C_{10}$ as minors. Thus, $\mathcal{O}\left[F_{7}\right] \subseteq \mathscr{E} x\left(M\left(K_{5}\right), C_{10}\right)$. It follows from (1) that the growth rate $g(n)$ of $\mathscr{E} x\left(M\left(K_{5}\right), C_{10}\right)$ equals 4 for $n \geqslant 3$.

This completes the proof of (2.6).

We turn now to the proof of (2.5). As in the earlier proof, we first prove

(8.21) THEOREM. Let $\mathscr{C}$ be a minor-closed class of binary geometries with maximum growth rate at least 4 . Then $\mathscr{C}$ contains $M\left(K_{5}\right)$ or $F_{7}$.

Sketch of Proof. Proceed as in the proof of (8.1) using Dirac's theorem (6.1) and (7.7). The case analyses are somewhat simpler.

The proof of (2.5) can now be completed by observing that the class $\mathcal{O}\left[M\left(K_{4}\right)\right]$, defined analogously to $\mathcal{O}\left[F_{7}\right]$, is contained in $\mathscr{E} x\left(M\left(K_{5}\right), F_{7}\right)$ and attains the maximum growth rate of 3 . 
9. Alternative proofs. Using Seymour's decomposition theory for regular geometries, we obtain an alternative proof for (2.5), and the following stronger version of (2.7).

(9.1) ThEOREM. Let $h(1)=1, h(n)=3 n-3$ if $n \geqslant 2$ and is even, and $h(n)=3 n$ -2 if $n \geqslant 2$ and is odd. Then $h(n)$ is the size function for $\mathscr{E} x\left(M\left(K_{5}\right), F_{7}^{*}\right)$.

However, (2.6) seems to be unobtainable from any similar decomposition theory. We shall assume that the reader is familiar (at least with the definitions and results) with [12 and 9].

Our most powerful tool is the following structure theorem for $\mathscr{E} x\left(F_{7}\right)$ and $\mathscr{E} x\left(F_{7}^{*}\right)$.

(9.2) Lemma. $A$ geometry not containing $F_{7}$ (respectively, $F_{7}{ }^{*}$ ) as a minor can be obtained by taking 0-sums, 1-sums, or parallel connections at a point of regular geometries and copies of $F_{7}^{*}$ (respectively, $F_{7}$ ). A regular geometry, in turn, can be obtained by taking 1-, 2-, or 3-sums of graphic or cographic geometries and copies of $R_{10}$

Proof. The first assertion follows from (i) Tutte's theorem [14 or 17, p. 175] stating that the class of regular geometries equals $\mathscr{E} x\left(F_{7}, F_{7}^{*}\right)$, and (ii) Seymour's lemma-or its dual-stating that $F_{7}{ }^{*}$ is a splitter for $\mathscr{E} x\left(F_{7}\right)((7.6)$ in [12]). The second assertion is the main result (14.3) of [12].

Since $M\left(K_{5}\right)$ is not cographic (see, for example, [17, p. 176]) and $R_{10}$ does not contain $M\left(K_{5}\right)$ as a minor (easy checking), a geometry in $\mathscr{E} x\left(M\left(K_{5}\right), F_{7}\right)$ (respectively, $\left.\mathscr{E} x\left(M\left(K_{5}\right), F_{7}^{*}\right)\right)$ can be obtained as in (9.2) with the additional requirement that the graphic geometries used do not contain $M\left(K_{5}\right)$ as a minor.

(9.3) Lemma. Let $k(1)=1$ and $k(n)=3 n-3$ for $n \geqslant 2$. Then $k(n)$ is the size function of the class of graphic geometries not containing $M\left(K_{5}\right)$ as a minor. The function $k(n)$ is also the size function of the class of cographic geometries.

Proof. The first assertion is a known result in extremal graph theory [10 or 1, p. 375]. Note that the rank of the cycle geometry of a connected graph is one less than the number of vertices.

Now let $M(\Gamma)^{*}$ denote the cocycle geometry of the graph $\Gamma$.

(9.3.1) Let $G$ be a connected cographic geometry. Then $G$ is isomorphic to $M(\Gamma)^{*}$ for a 3-connected graph $\Gamma$.

Proof. Among all the graphs $\Delta$ for which $M(\Delta)^{*} \cong G$, choose one, $\Gamma$, with the smallest number of vertices. As $G$ is connected as a geometry, $\Gamma$ is 2-connected (see [19 or 17, p. 69]). Suppose that $\Gamma$ can be disconnected by removing two edges $\alpha$ and $\beta$. These two edges form a two-element circuit or double point in the cocycle matroid of $\Gamma$, and hence one of them, $\alpha$ say, can be deleted without changing the cocycle geometry $M(\Gamma)^{*}$. Deletions in the cocycle matroid correspond to contractions in $\Gamma$. Thus, we obtain a graph $\Gamma / \alpha$ with $M(\Gamma / \alpha)^{*} \cong G$, having one fewer vertex than $\Gamma$, a contradiction. 
By (9.2.1), it suffices to prove the second assertion for cographic geometries of the form $M(\Gamma)^{*}$, where $\Gamma$ is 3 -connected. But this follows from equation (i) in $[9, \mathrm{p}$. 211].

The proof of (2.5) can now be completed with three easy observations. Let $G_{1}$ and $G_{2}$ be geometries such that $\left|G_{i}\right| \leqslant k\left(\operatorname{rank}\left(G_{i}\right)\right)$ for $i=1$ and 2 . Let $G$ be the 1 -sum, 2-sum, 3-sum, or parallel connection at a point of $G_{1}$ and $G_{2}$. Then, as $k$ is an affine function, $|G| \leqslant k(\operatorname{rank}(G))$. Moreover, both $F_{7}^{*}$ and $R_{10}$ have size less than the size specified by $k(n)$. Hence, we conclude that a geometry of rank $n$ in $\mathscr{E} x\left(M\left(K_{5}\right), F_{7}\right)$ has size at most $k(n)$. The maximum size $k(n)$ can be attained by, say, the cycle geometry of a maximal planar graph.

(9.1) can be proved similarly. For $n$ even, the maximum size $h(n)$ can be attained by taking parallel connections at a point of copies of $F_{7}$. (It is easy to check that these geometries do not contain $M\left(K_{5}\right)$ or $F_{7}{ }^{*}$ as a minor.)

10. A conjecture about a maximum class. To state our conjecture, we need an easy fact.

(10.1) LEMMA. Let $\mathscr{C}$ and $\mathscr{D}$ be minor-closed classes of geometries with the same size function $h(n)$. Then their set-theoretic union $\mathscr{C} \cup \mathscr{D}$ is also a minor-closed class with size function $h(n)$.

Let $h(n)$ be the size function of a minor-closed class. The maximum class $\mathscr{M}$, with size function $h(n)$ is the union of all the minor-closed classes with size function $h(n)$. The maximum class $\mathscr{M}$ has the property: if $\mathscr{C}$ is a minor-closed class with size function $h(n)$, then $\mathscr{C} \subseteq \mathscr{M}$.

(2.5) and (5.1) can be restated in terms of maximum classes.

(10.2) TheOREM. (a) Let $h(1)=1$ and $h(n)=2 n-1$ for $n \geqslant 2$. Then $\mathscr{E} x\left(M\left(K_{4}\right)\right)$ is the maximum class with size function $h(n)$.

(b) Let $h(1)=1$ and $h(n)=3 n-3$ for $n \geqslant 2$. Then $\mathscr{E} x\left(M\left(K_{5}\right), F_{7}\right)$ is the maximum class with size function $h(n)$.

(10.2) follows from the observation that the excluded minors have size strictly greater than the size specified by the size function.

(10.2) also indicates that (2.5) and (5.1) are the best results possible. This is not true of (2.6) and leads to the following conjecture.

(10.3) Conjecture. Let $h(1)=1$ and $h(n)=4 n-5$ for $n \geqslant 2$. Then the maximum class with size function $h(n)$ is $\mathscr{E} x\left(M\left(K_{6}\right), C_{12}, D_{12}\right)$.

Here, $C_{12}$ and $D_{12}$ are the rank 4 binary geometries defined in (7.6). Strong evidence for this conjecture comes from a result in extremal graph theory.

(10.4) THEOREM [8 OR 1, P. 373]. Let $\Gamma$ be a graph in which every vertex has degree at least 4. Then $\Gamma$ contains as a subcontraction $K_{5}$ or $K_{2,2,2}$.

Here, $K_{2,2,2}$ is the complete tripartite graph on three sets of vertices, each of size 2. The relevance of (10.4) to (10.3) is due to: (a) if a bond graph $\Gamma(x, G)$ is a $K_{5}$, 
then $G$ is isomorphic to $M\left(K_{6}\right)$ or $D_{12}$, and (b) if a bond graph $\Gamma(x, G)$ is a $K_{2,2,2}$ and $G$ has rank 4 , then $G$ is isomorphic to $C_{12}$. Using (10.4) to settle (10.3) would entail developing techniques to use results about subcontractions (rather than results about topological subgraphs) from extremal graph theory to solve growth rate problems. Such a development would be of great interest in its own right.

ACKNOWLEDGement. The author would like to thank Jeff Kahn for many conversations on the subject of this paper.

\section{REFERENCES}

1. B. Bollobás, Extremal graph theory, Academic Press, London and New York, 1978.

2. T. Brylawski, Modular constructions for combinatorial geometries, Trans. Amer. Math. Soc. 203 (1975), 1-44.

3. T. H. Brylawski and D. Lucas, Uniquely representable combinatorial geometries, Colloq. Internaz. sulle Teorie Combinatorie (Roma, 1973), Atti dei Convegni Lincei 17, Tomo I, Accad. Naz. Lincei, Rome, 1976, pp. 83-104.

4. H. H. Crapo and G.-C. Rota, On the foundations of combinatorial theory: Combinatorial geometries (prelim. ed.), M. I. T. Press, Cambridge, Mass., 1970.

5. G. A. Dirac, In abstrakten Graphen vorhandene vollständige 4-Graphen und ihre Unterteilungen, Math. Nachr. 22 (1960), 61-85.

6. R. J. Duffin, Topologv of series-parallel networks, J. Math. Anal. Appl. 10 (1965), 303-318.

7. J. Edmonds and D. R. Fulkerson, Transversals and matroid partitions, J. Res. Nat. Bur. Standards Sect. B 69 (1965), 147-153.

8. R. Halin and H. A. Jung, Üher Minimalstrukturen von Graphen, insbesondere von $n$-fach zusammenhängenden Graphen, Math. Ann. 152 (1963), 75-94.

9. F. Jaeger, Flows and generalized coloring theorems in graphs, J. Combin. Theory Ser. B 26 (1979), 205-216.

10. W. Mader, Homomorphieeigenschaften und mitlere Kantendichte von Graphen, Math. Ann. 174 (1967), 265-268.

11. J. Pelikan, Valency conditions for the existence of certain subgraphs, Theory of Graphs (Proc. Colloq., Tihany, 1966), Academic Press, New York, 1968, pp. 251-258.

12. P. D. Seymour, Decomposition of regular matroids, J. Combin. Theory Ser. B 28 (1980), 305-359.

13. C. Thomassen, Some homeomorphism properties of graphs, Math. Nachr. 64 (1974), 119-133.

14. W. T. Tutte, A homotopy theorem for matroids. I and II, Trans. Amer. Math. Soc. 88 (1958), $144-176$.

15. __ Matroids and graphs, Trans. Amer. Math. Soc. 90 (1959), 527-552.

16. P. N. Walton and D. J. A. Welsh, On the chromatic number of binary matroids, Mathematika 27 (1980), 1-9.

17. D. J. A. Welsh, Matroid theory, Academic Press, London and New York, 1976.

18. N. L. White (ed.), Combinatorial geometry, Cambridge Univ. Press, Cambridge, 1985.

19. H. Whitney, Non-separable and planar graphs, Trans. Amer. Math. Soc. 34 (1932), 339-362.

Department of Mathematics, North Texas State University, Denton, Texas 76203 